\title{
Microscopic Theory of Pion Production and Sidewards Flow in Heavy-Ion Collisions
}

\author{
H. Kruse, ${ }^{\text {(a) }}$ B. V. Jacak, and H. Stöcker \\ Department of Physics and Astronomy, and National Superconducting Cyclotron Laboratory, \\ Michigan State University, East Lansing, Michigan 48824 \\ (Received 9 July 1984)
}

\begin{abstract}
Nuclear collisions from 0.3 to $2 \mathrm{GeV} /$ nucleon are studied in a microscopic theory based on Vlasov's self-consistent mean field and Uehling-Uhlenbeck's two-body collision term which respects the Pauli principle. The theory explains simultaneously the observed collective flow and the pion multiplicity and gives their dependence on the nuclear equation of state.
\end{abstract}

PACS numbers: $25.70 .-\mathrm{z}$

One of the most intriguing motivations for studying relativistic nucleus-nucleus collisions is the unique opportunity to explore compressed and excited nuclear matter in the laboratory. A signature of compression is the collective sidewards flow predicted theoretically by nuclear fluid dynamics ${ }^{1}$ and classical microscopic many-body simulations. ${ }^{2}$ Recently, the predicted collective sidewards flow has been observed in highmultiplicity selected collisions of heavy nuclei. ${ }^{3,4}$ Another observable compression effect predicted by fluid dynamics is the dependence of the pion multiplicity on the nuclear compression energy at high densities. ${ }^{5}$ The pion multiplicities have been measured for near-central collisions of $\mathrm{Ar}(0.3-1.8 \mathrm{GeV} /$ nucleon) $+\mathrm{KCl}^{6}$

Both data sets present a challenge to microscopic theories: The flow calculations ${ }^{3,4}$ done with the standard intranuclear-cascade programs $\mathrm{s}^{7,8}$ result in forward-peaked angular distributions, in contrast to the data, and the calculated pion multiplicities ${ }^{6-8}$ drastical- ly overestimate the experimental yields. These large discrepancies are surprising in view of the success of the cascade model in describing inclusive data. ${ }^{7,8}$ It has been conjectured ${ }^{6}$ that the difference between measured pion yields and cascade predictions is due to the neglect of compression energy in the cascade approach and thus may be used to extract the nuclear equation of state at high densities.

In this Letter we present a microscopic theory which explains for the first time simultaneously both the observed collective flow and the pion multiplicity and gives their dependence on the nuclear equation of state. Our approach is based on Vlasov's equation for the evolution of the single-particle distribution function $f$ of a collisionless plasma in a self-consistent mean potential field supplemented by UehlingUhlenbeck's quantum mechanical extension of Boltzmann's two-body collision term which respects the Pauli principle. This extended Boltzmann equation can be written 9,10

$$
\begin{aligned}
\frac{\partial f}{\partial t}+\mathbf{v} \cdot \frac{\partial f}{\partial \mathbf{r}}+ & \frac{\partial U(n)}{\partial \mathbf{r}} \cdot \frac{\partial f}{\partial \mathbf{v}} \\
& =\int \frac{d^{3} p_{2} d^{3} p_{1}^{\prime} d^{3} p_{2}^{\prime}}{(2 \pi)^{6}} \sigma v_{12}\left[f f_{2}\left(1-f_{1}^{\prime}\right)\left(1-f_{2}^{\prime}\right)-f_{1}^{\prime} f_{2}^{\prime}(1-f)\left(1-f_{2}\right)\right] \delta^{3}\left(p+p_{2}-p_{1}^{\prime}-p_{2}^{\prime}\right) .
\end{aligned}
$$

The Vlasov equation is solved by simultaneous numerical integration of the classical equations of motion of fifteen parallel ensembles of $A_{P}+A_{T}$ test particles, which are initially assigned Fermi momenta and random positions in a sphere of nuclear radius. The ensemble-averaged phase-space density is computed at each synchronization time step in a six-dimensional sphere around each test particle. This ensemble averaging ${ }^{9,10}$ ensures a reasonably smooth singleparticle distribution function $f(r, p, t)$ which is used to determine the mean field $U(n)$ and the Pauli-blocking probability $(1-f)(1-f)$.
Two test particles from a given ensemble may undergo $s$-wave scattering if they approach each other within a distance $d^{2}=\sigma / \pi$ and the resulting final states are not Pauli blocked. Here $\sigma$ denotes the experimental scattering cross section of protons, neutrons, and pions. The experimental inelastic-scattering cross sections are used to calculate pion production and absorption through the delta resonance.

Three different Skyrme parametrizations have been chosen to represent the mean field $U$ due to a stiff (compression constant $K=375 \mathrm{MeV}$ ), a medium ( $K=200 \mathrm{MeV})$, and a supersoft nuclear equation of state:

$$
\begin{aligned}
& U(n)=-124 n / n_{0}+70.5\left(n / n_{0}\right)^{2} \mathrm{MeV} \quad(K=375 \mathrm{MeV}), \\
& U(n)=-356 n / n_{0}+303\left(n / n_{0}\right)^{7 / 6} \mathrm{MeV} \quad(K=200 \mathrm{MeV}) .
\end{aligned}
$$


The supersoft potential equals (2a) at $n<n_{0}$, and is constant for $n>n_{0}$. It allows the study of bound nuclei with zero compression energy. $U$ is directly related to the nuclear equation of state via $U(n)$ $=\partial n(E) / \partial n$.

We have extensively tested the present method and the newly developed computer program. ${ }^{10}$ First, inclusive spectra of protons emitted from high-energy heavy-ion reactions have been calculated. They compare well with recent data. ${ }^{10,11}$ Second, we have studied proton-induced pion production on nuclear targets. Figure 1 shows the target-mass dependence of the observed $\pi^{+}$and $\pi^{-}$yields ${ }^{12}$ in comparison with the present theory. The absolute yields, the target-mass dependence, and the large (factors of 5) difference between the $\pi^{+}$and $\pi^{-}$yields as well as the pion spectra (not shown) are well reproduced.

The present approach has further been tested by turning off the Pauli blocking and mean potential field. Then the parallel ensembles decouple, and the test particles move on straight-line trajectories until they scatter. Thus the conventional intranuclear cascade model is recovered. The thus obtained "cascade nuclei" are unstable and expand as a result of the Fermi motion. ${ }^{8}$ It has been shown ${ }^{13}$ that this artificial expansion causes a rapid decrease of the density and drastically reduces the number of $n-n$ collisions if massive nuclei $(A-100)$ are studied. The artificial expansion of the cascade nuclei can be prevented by "freezing",13 the Fermi momenta of the nucleons or-as done in the present work - by use of a binding potential. The supersoft equation of state can be used to study binding without introducing compressional energy explicitly into the calculation. In order to simulate the standard cascade calculations as closely as possible, we

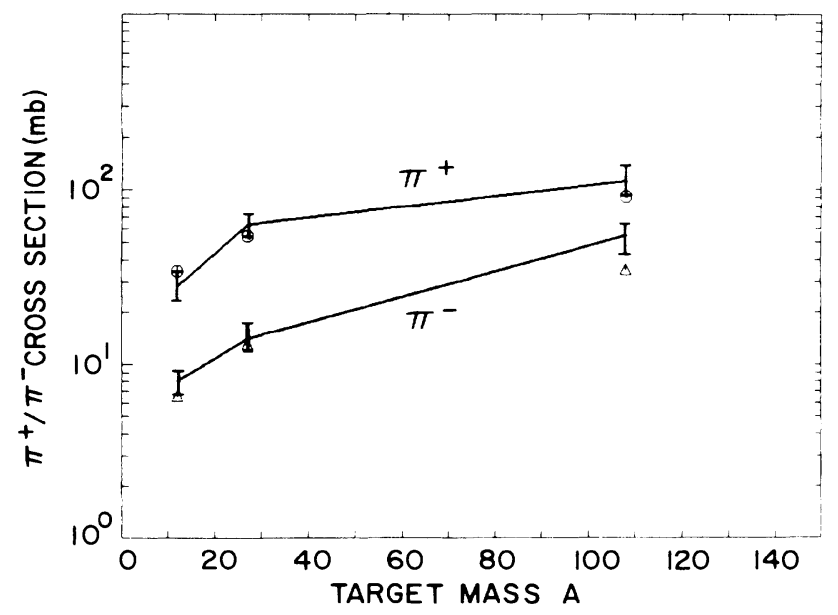

FIG. 1. Pion yields from $730-\mathrm{MeV}$ protons as a function of the target mass (Ref. 12). The lines and statistical error bars refer to the present theory. have used in our "cascade mode" Cugnon's "Pauli principle" prescription ( $n$ - $n$ collisions with c.m. energies below $50 \mathrm{MeV} /$ nucleon are inhibited). The pion yields calculated with the present program in the "cascade mode" (i.e., with the simplified Pauli blocker and no field) agree quantitatively with the cascade results. $^{6,8}$ Both results differ substantially from the results obtained with the present theory which uses a nuclear equation of state and phase-space Pauli blocking:

Take the 360-MeV/nucleon data, for instance: The negative pion yield is 1.05 in the "cascade mode," but drops by a factor of 2-to 0.56 - if the compression energy ( $2 a)$ is included - the suggested large influence of the nuclear matter equation of state 5,6 is observed. The pion yield drops further (to 0.46) when the Uehling-Uhlenbeck Pauli blocking is applied. These numbers differ by factors of 3 from results published in Ref. 9, where a method similar to the present one has been used to solve the the Boltzmann equation. A revised version of that program now reproduces our results. We would like to refer to other papers on pion production which use different approaches. ${ }^{14-17}$

The pion multiplicities are shown in Fig. 2 as a function of the bombarding energy. The present theory with equation of state (2a) plus phase-space Pauli blocker compares well with the data, ${ }^{6}$ while our "cascade mode" overestimates the pion yields by factors $>2$ at energies up to $1 \mathrm{GeV} /$ nucleon, just like the cascade calculations. ${ }^{6-8}$ The drop in the pion yield is found to be due to the transformation of kinetic energy into potential energy during the high-density phase of the reaction as well as due to Pauli blocking. To

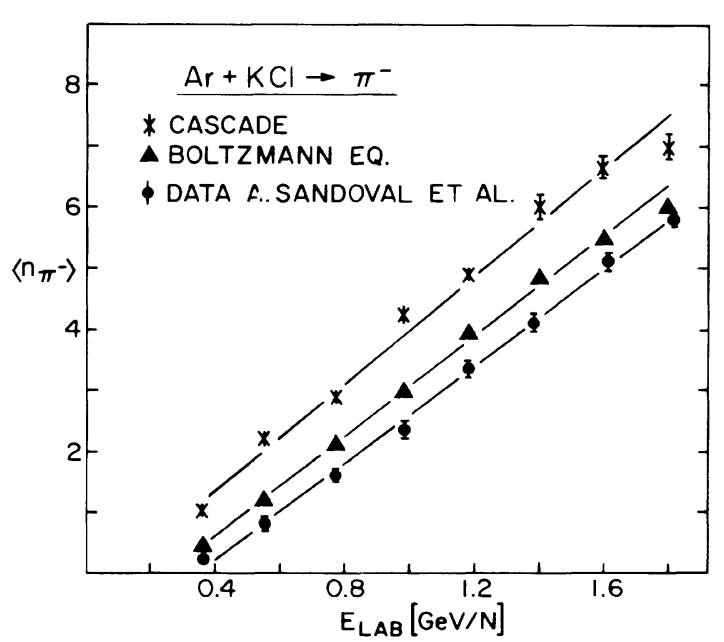

FIG. 2. Pion multiplicity for central collisions $(b<2.4$ $\mathrm{fm}$ ) of $\mathrm{Ar}+\mathrm{KCl}$. The data (Ref. 6, circles) are compared to the present theory in the "cascade mode" (crosses) and to the same theory with compression energy and phase-space Pauli blocking included (triangles). 
check the sensitivity of the pion yields to the equation of state, the calculations have been repeated with the medium potential (2b). At $772 \mathrm{MeV} /$ nucleon we find $\left\langle\pi^{-}\right\rangle=2.45 \pm 0.09$ and $2.13 \pm 0.07$ with the medium and the stiff equation of state, respectively. At lower energies, statistical error bars of $15 \%$ preclude an accurate assessment of the influence of the potential. At all other energies, where the statistical error bars are $3 \%$, the yields are systematically higher (by about $10 \%$ ) with the medium equation of state than with the stiff equation of state. For the time being we feel unable, though, to extract a nuclear equation of state from the data. ${ }^{6}$

We have also investigated the observed sidewards peaking [Fig. 3(b)] in multiplicity selected $\mathrm{Nb}(400$

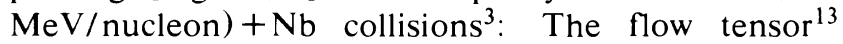
$F_{i j}=\Sigma_{\nu} p_{i} p_{j} / 2 m_{\nu}$ is determined on an event-by-event basis and the direction of the maximum kinetic-energy flow is determined. Cascade calculations produce forward-peaked distributions. ${ }^{3,13}$ We would like to emphasize again the important role of the binding potential: If the binding potential is neglected as in Cugnon's original program, 8,13 finite flow angles may be obtained ${ }^{18}$ as a result of the sidewards expansion of the unbound projectile and target nucleons. This is demonstrated in Fig. 3(c), which shows the flow-angle distributions obtained with the original Cugnon program $^{8}$ (i.e., unstable nuclei) and the modified version $^{6,13}$ of his code with "frozen" Fermi momenta.

Figure 3(a) shows the flow-angle distribution for $\mathrm{Nb}(400 \mathrm{MeV} /$ nucleon $)+\mathrm{Nb}$ at impact parameter $b=1$ $\mathrm{fm}$ with the medium and the stiff equation of state. Observe the strong sidewards maximum at large angles in both cases and the drastic influence of the equation of state: The average flow angle is $43^{\circ}$ with the stiff equation of state, while it is $28^{\circ}$ with the medium one. As a consequence of this substantial variation of the flow with the nuclear potential, it seems feasible to extract the nuclear equation of state from a comparison of the data with high-statistics calculations, whence the experimental trigger conditions and efficiency cuts are carefully simulated in the calculations.

In conclusion, a microscopic theory which incorporates both a self-consistent mean field and two-body collisions which respect the Pauli principle explains both pion multiplicity and collective flow observed in central nucleus-nucleus collisions. The results exhibit a significant influence of both the nuclear equation of state and the Pauli principle.

Discussions with J. Aichelin, G. Bertsch, J. Harris, and R. Stock are acknowledged. This work was supported by the National Science Foundation.

(a) Present address: Telco Research, Nashville, Tenn. 37203.

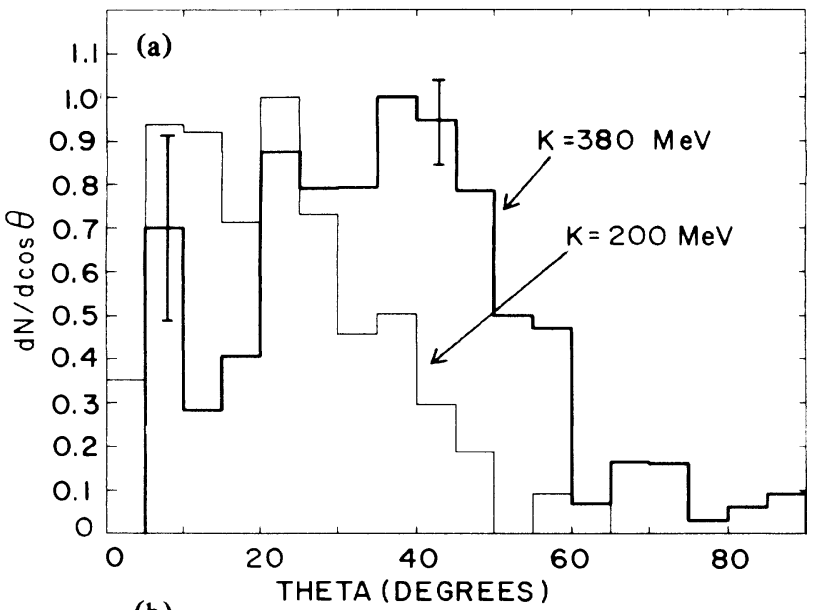

(b)

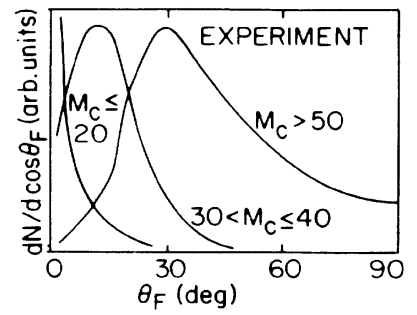

(c)

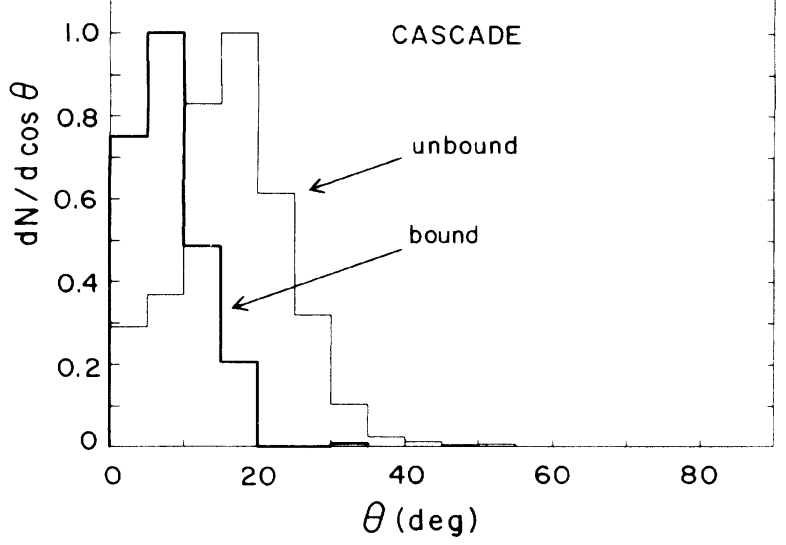

FIG. 3. Flow-angle distributions $d N / d \cos \theta_{F}$ for ${ }^{93} \mathrm{Nb}(400$ $\mathrm{MeV} /$ nucleon $)+{ }^{93} \mathrm{Nb}$ : (a) The present theory with the medium and hard equation of state; if the Pauli principle is neglected, the maximum in the angular distribution shifts to larger angles (by about $20^{\circ}$ ). (b) "Plastic-ball" data (Ref. 3) for various multiplicity bins. (c) Results obtained with the standard cascade with use of unstable nuclei (Ref. 8) and the "frozen" Fermi-momenta approximation (Ref. 13).

IW. Scheid, H. Müller, and W. Greiner, Phys. Rev. Lett. 32, 741 (1974); H. Stöcker, J. A. Maruhn, and W. Greiner, Phys. Rev. Lett. 44, 725 (1980); J. I. Kapusta and D. Strottman, Phys. Lett. 103B, 269 (1981); G. Buchwald, G. Graebner, J. Theis, J. A. Maruhn, W. Greiner, and H. Stöcker, Phys. Rev. Lett. 52, 1594 (1984), and Phys. Rev. C 28, 2349 (1983). 
2J. J. Molitoris, J. B. Hoffer, H. Kruse, and H. Stöcker, Phys. Rev. Lett. 53, 899 (1984); R. Malfliet, University of Groningen, Kernfysisch Versneller Institute Report, 1984 (to be published); S. M. Kiselev, Institut of Theoretical and Experimental Physics Report No. ITEP-123, 1984 (unpublished). Transverse peaking of cross sections and a mass dependence, which is not obtained from hydrodynamics, has been predicted for central collisions in earlier many-body simulations: A. R. Bodmer, C. N. Panos, and A. D. MacKellar, Phys. Rev. C 22, 1025 (1980); see also E. Halbert, Phys. Rev. C 23, 295 (1981).

${ }^{3}$ H. A. Gustafsson, H. H. Gutbrod, B. Kolb, H. Löhner, B. Ludewigt, A. M. Poskanzer, T. Renner, H. Riedesel, H. G. Ritter, A. Warwick, F. Weik, and H. Weiman, Phys. Rev. Lett. 52, 1590 (1984).

${ }^{4}$ R. E. Renfordt, D. Schall, R. Bock, R. Brockmann, J. W. Harris, A. Sandoval, R. Stock, H. Stroebele, D. Bangert, W. Rauch, G. Odyniec, H. G. Pugh, and L. S. Schroeder, Phys. Rev. Lett. 53, 763 (1984); D. Keane et al., to be published.

${ }^{5}$ H. Stöcker, W. Greiner, W. Scheid, Z. Phys. A 286, 121 (1978); P. Danielecwicz, Nucl. Phys. A314, 465 (1979); H. Stöcker, J. Phys. Lett. 10, 111 (1984).

${ }^{6}$ R. Stock, R. Bock, R. Brockmann, J. W. Harris, A. Sandoval, H. Stroebele, K. L. Wolf, H. G. Pugh, L. S. Schroeder, M. Maier, R. E. Renfordt, A. Dacal, and M. E. Ortiz, Phys. Rev. Lett. 49, 1236 (1982); A. Sandoval et al., Phys. Rev. Lett. 45, 874 (1980); J. W. Harris et al., to be published.

${ }^{7}$ Y. Yariv and Z. Fraenkel, Phys. Rev. C 20, 2227 (1979), and C 24, 488 (1981); V. D. Toneev and K. K. Gudima,
Phys. A400, 173 (1983).

$8 \mathrm{~J}$. Cugnon, D. Kinet, and J. Vandermeulen, Nucl. Phys. A379, 553 (1982); J. Cugnon, T. Mituzani, and J. Vandermeulen, Nucl. Phys. A352, 505 (1981)

${ }^{9}$ G. Bertsch, H. Kruse, and S. Das Gupta, Phys. Rev. C 29, 673 (1984).

${ }^{10} \mathrm{H}$. Kruse, B. V. Jacak, and H. Stöcker, unpublished.

${ }^{11}$ S. Nagamiya, M. C. Lemaire, E. Moeller, S. Schnetzer, G. Shapiro, H. Steiner, and I. Tanihata, Phys. Rev. C 24, 971 (1981); B. Jacak, G. D. Westfall, C. K. Gelbke, L. H. Harwood, W. G. Lynch, D. K. Scott, H. Stöcker, M. B. Tsang, and T. J. M. Symons, Phys. Rev. Lett. 51, 1846 (1984).

${ }^{12}$ D. R. F. Cochran, P. N. Dean, P. A. M. Gram, E. A. Knapp, E. R. Martin, D. E. Nagle, R. B. Perkins, W. J. Shlaer, H. A. Thiessen, and E. D. Theriot, Phys. Rev. D 6 , 3085 (1972).

${ }^{13}$ M. Gyulassy, K. A. Fraenkel, and H. Stöcker, Phys. Lett. 110B, 185 (1982).

14I. Montvay, and J. Zimanyi, Nucl. Phys. A312, 290 (1979).

${ }^{15}$ R. Malfliet and B. Schürmann, Phys. Rev. C 28, 1136 (1983).

${ }^{16}$ M. Cahay, J. Cugnon, and J. Vandermeulen, Nucl. Phys. A411, 524 (1983).

${ }^{17}$ Y. Kitazoe, M. Gyulassy, P. Danielewicz, H. Toki, Y. Yamamura, and M. Sano, Phys. Lett. 138B, 341 (1984); Phys. Rev. C 29, 828 (1984). This cascade version assumes a zero resonance lifetime and neglects pion absorption, which results in largely different pion yields (Ref. 8).

$18 \mathrm{~J}$. Cugnon, and D. L'Hote, unpublished. 